\title{
An analysis of national data on care-seeking behaviour by parents of children with suspected pneumonia in Nigeria
}

\author{
M B Abdulkadir, ${ }^{1}$ MBBS, FWACP (Paed); Z A Abdulkadir, ${ }^{2}$ MBBS, MWACS; W B R Johnson, ${ }^{1}$ MBBS, FWACP (Paed) \\ ${ }^{1}$ Department of Paediatrics and Child Health, University of Ilorin/University of Ilorin Teaching Hospital, Ilorin, Kwara State, Nigeria \\ ${ }^{2}$ Department of Obstetrics and Gynaecology, University of Ilorin Teaching Hospital, Ilorin, Kwara State, Nigeria
}

Corresponding author: M B Abdulkadir (docmohng@gmail.com)

\begin{abstract}
Background. Pneumonia is responsible for 940000 under-5 deaths annually. Most of these deaths result from delays in instituting effective treatment.

Objectives. To determine care-seeking behaviour by parents of children with pneumonia and sociodemographic factors that influence decisions to seek appropriate care.

Methods. The study was an analysis of the Nigeria Demographic and Health Survey 2013, which was a nationwide cross-sectional survey using a stratified cluster design of 40680 households. All children under-5 living in the surveyed households with suspected pneumonia in the preceding 2 weeks were recruited along with their mothers. Sociodemographic characteristics of the parents and where they sought care for their child were obtained. Binomial logistic regression analysis was used to determine the contribution of various sociodemographic variables to the decision on seeking appropriate medical care.

Results. Of the 28950 children surveyed, 565 had suspected pneumonia, which equates to an occurrence rate of 19.5 per 1000 children. About $36 \%$ of parents were judged to have sought appropriate care when their children had pneumonia. High paternal education, health decision-making by both husband and wife, and belonging to the higher quintiles on a wealth index were factors that positively influenced care-seeking behaviour. Conclusion. Care-seeking behaviour for pneumonia is poor. Paternal education and joint decision-making are key determinants of parents seeking appropriate care for their children with suspected pneumonia in Nigeria.
\end{abstract}

S Afr J Child Health 2016;10(1):92-95. DOI:10.7196/SAJCH.2016.v10i1.1076

Acute respiratory infections are a leading cause of death in children $<5$ years old, responsible for 940000 (15\%) of all deaths in 2013. ${ }^{[1]}$ There has been a $44 \%$ decline in pneumonia-related deaths since 2000. ${ }^{[1]}$ This decline has been more pronounced in developed countries and south Asia compared with sub-Saharan Africa. ${ }^{[2,3]}$ About half the world's pneumonia-related deaths occur in Nigeria, the Democratic Republic of the Congo, Ethiopia, India and Pakistan ${ }^{[2]}$ Several strategies have been developed globally to address pneumonia-related mortality. These strategies have been encapsulated in the Global Action Plan for the Prevention and Control of Pneumonia (GAPP).$^{[4,5]}$ Early diagnosis and prompt treatment with appropriate antibiotics have been critical in addressing such mortality. ${ }^{[2]}$ This may be deployed as community or facility-based interventions. The case management approach formulated by the World Health Organization (WHO) has as its main foundations: appropriate use and choice of antibiotics against the major causes of bacterial pneumonia (pneumococcus and Hib); training health workers to utilise simple clinical signs to assess severity and guide appropriate treatment; and appropriate and effective use of oxygen. ${ }^{[5]}$

Launched in 2009, GAPP recognised the importance of timely treatment and set an ambitious target of ensuring that $90 \%$ of children with pneumonia have access to appropriate pneumonia case management by $2015 .{ }^{[6]}$ Unfortunately, in most sub-Saharan African countries, parents often seek healthcare from sources that lack the requisite knowledge, skills or facilities to provide appropriate care ${ }^{[7-9]}$ This has a significant effect on pneumonia morbidity and mortality. ${ }^{[10]}$ Studies have shown that the care-seeking behaviour of parents is influenced by household size, age, education of the mother, past experiences, traditional beliefs, cost and location of health facilities. ${ }^{[7,11-13]}$ West and Central African regional data have demonstrated that appropriate care-seeking behaviour for pneumonia has improved from 34 to $39 \%$ between 2000 and 2013. ${ }^{[10]}$
Despite this modest increase, there have been wide disparities within regions, countries and individual states. ${ }^{[10]}$ It thus becomes crucial to generate local data regarding appropriate care-seeking behaviour to guide researchers and policy makers on effective pneumonia case management. The study aimed to determine care-seeking behaviours of parents of children with a symptom complex of pneumonia and factors that may influence these behaviours.

\section{Methods}

The study utilised the publicly available dataset of the Nigeria Demographic and Health Survey (NDHS) 2013, conducted by the National Population Commission (NPC). Permission was obtained for the use of the data from the Demographic and Health Surveys (DHS) Programme and Inner City Fund International. A detailed description of the study design, participants and study instruments has been previously published by the NPC. ${ }^{[14]}$ Ethical approval for the survey was obtained from the National Health Research Ethics Committee. Informed consent was obtained from study participants before they were allowed to participate in the survey. The data contained no information that could be used to trace participants.

A nationwide cross-sectional survey was carried out and a stratified three-stage cluster design was adopted. ${ }^{[14]}$ The first stage involved the selection of 893 localities with probability of selection being proportional to size. At least one enumeration area (defined from the last population census) was randomly selected from each of the localities. This resulted in 904 enumeration areas being selected. In a few of the larger localities, more than one enumeration area was selected. ${ }^{[14]}$ A household listing was made for each enumeration area. Forty-five households were selected in urban and rural clusters using systematic probability sampling with the sampling frame being the list of households in each enumeration area. ${ }^{[14]}$ 
A total of 40680 households were surveyed for the demographic and health survey. Trained interviewers administered a questionnaire to each household with questions pertaining to household characteristics, health of children and care-seeking behaviour. ${ }^{[14]}$ Children aged 0 59 months who had a cough and chest-related fast or difficult breathing in the preceding two weeks were recruited into the study alongside their parents. These symptoms constituted 'suspected pneumonia' as defined by the WHO's Integrated Management of Childhood Illnesses. ${ }^{[2,15]}$ Appropriate care-seeking was defined as seeking care from all public or private hospitals, health centres or posts, private doctors and community health workers. It excludes no medical care, pharmacies, shops and traditional practitioners. ${ }^{[2,10]}$ Data analysis was carried out using SPSS version 20 software (IBM, USA). Frequencies of missing variables were indicated, where present, if they constituted more than $1 \%$ of the overall proportion of the variable. However, these missing variables were excluded from $\chi^{2}$ and regression analyses as recommended by the DHS Programme. ${ }^{[16]}$ Data were weighted using sampling weights provided within the database. Sampling weights are adjustment factors applied to each case in tabulations to adjust for differences in probability of selection and interview between cases in a sample, either due to design or chance. ${ }^{[16]}$ Often in DHS the samples are selected to ensure the maximum representation of certain variables, such as urban/rural setting, which introduces bias into the sample selection process. To eliminate this bias, the frequency of occurrence of each event is weighted to indicate the number of individuals that should have been selected had bias not been present in the sample. ${ }^{[16]}$ Wealth index was generated by principal component analysis of household assets. The sum of scores obtained for each household, standardised to a normal distribution and appropriate cut-offs, was used to define poorest, poorer, middle, higher and highest quintiles. ${ }^{[17]}$ Frequency distribution tables and cross-tabulation of variables were generated. Mean and standard deviation for continuous variables were provided and the proportion for categorical variables was also determined. Basic tests of statistical significance such as $\chi^{2}$ and Student $t$-tests were utilised as required. Maternal, household, socioeconomic, education and child factors were compared individually to care-seeking categories through univariate analysis. Significant factors were built into a multiple logistic regression model that was used to identify significant predictors of appropriate care-seeking behaviour. Unweighted data were used for the regression analysis as recommended by the DHS Programme. ${ }^{[16]}$ Odds ratio and $p$-value were used to determine significance of associations. $P<0.05$ was considered significant.

Table 1. Univariate analysis of weighted numbers and percentages of selected determinants of appropriate care-seeking behaviour

\begin{tabular}{|c|c|c|c|}
\hline Variables & $\begin{array}{l}\text { Appropriate, } \\
n(\%)^{*}\end{array}$ & $\begin{array}{l}\text { Inappropriate, } \\
n(\%)^{\star}\end{array}$ & $p$-value \\
\hline Age of child (months), mean (SD) & $24.82(14.78)$ & $22.62(15.39)$ & 0.110 \\
\hline Age of mother (years), mean (SD) & $28.88(7.02)$ & $28.81(7.08)$ & 0.920 \\
\hline Age of household head (years), mean (SD) & $39.70(10.36)$ & $38.75(10.14)$ & 0.310 \\
\hline Number of children under five in household & $2.20(1.33)$ & $2.32(1.25)$ & 0.290 \\
\hline Sex of child & $n=194$ & $n=350$ & 0.360 \\
\hline Male & $101(52.1)$ & $168(48.0)$ & \\
\hline Female & 93 (47.9) & $182(52.0)$ & \\
\hline Place of residence & $n=195$ & $n=350$ & $0.0001^{\dagger}$ \\
\hline Urban & $72(36.9)$ & $77(22.0)$ & \\
\hline Rural & $123(63.1)$ & $273(78.0)$ & \\
\hline Highest maternal educational level & $n=195$ & $n=349$ & $0.001^{\dagger}$ \\
\hline None & $86(44.1)$ & $183(52.4)$ & \\
\hline Primary & $32(16.4)$ & $81(23.2)$ & \\
\hline Secondary & $64(32.8)$ & $79(22.6)$ & \\
\hline Tertiary & $13(6.7)$ & $6(1.7)$ & \\
\hline Duration of mother education (years), mean (SD) & $5.32(5.52)$ & $3.57(4.41)$ & $0.0001^{\dagger}$ \\
\hline Highest paternal educational level & $n=187$ & $\boldsymbol{n}=328$ & $0.0001^{\dagger}$ \\
\hline None & $68(36.4)$ & $149(45.4)$ & \\
\hline Primary & $29(15.5)$ & $72(22.0)$ & \\
\hline Secondary & $57(30.5)$ & $90(27.4)$ & \\
\hline Tertiary & $33(17.6)$ & $17(5.2)$ & \\
\hline
\end{tabular}

\section{Results}

There were 28950 children who were younger than 60 months of age in the 40680 households surveyed. Of these, 14509 (50.1\%) were males and $14440(49.9 \%)$ were females. The mean (standard deviation (SD)) age of the children was 28.1 (17.3) months with a range of 0 - 59 months. Of the 28950 under-5 children, 565 (2.0\%; $95 \%$ confidence interval (CI) 1.8 - 2.1) fulfilled the criteria for suspected pneumonia in the preceding 2 weeks. This meant the pneumonia prevalence rate was 19.5 per 1000 children. Among the children with suspected pneumonia, there were 279 (49.4\%) males. The mean (SD) age of children with suspected pneumonia was 23.5 (15.2) months with a range of 0 - 59 months. Regarding the age distribution of the children with pneumonia, of the total number of children: 137 (24.3\%) were aged between 0 and 11 months; 183 (32.4\%) were aged between 12 and 23 months; 115 (20.4\%) were aged between 24 and 35 months; 57 (10.0\%) were aged between 36 and 47 months; and $64(11.3 \%)$ were aged between 48 and 59 months.

There were 544 valid responses to care seeking for the children (21 cases had missing records regarding care for the child and were excluded from subsequent analyses of careseeking behaviour). A total of 194 (35.7\%) parents sought appropriate care when their child had suspected pneumonia. Selected sociodemographic factors were compared with care-seeking behaviour for pneumonia in a univariate analysis using weighted data to account for probability of sampling (Tables 1 and 2). Factors that were significantly associated with care-seeking behaviour for suspected pneumonia were: living in an urban setting $(p=0.001)$; maternal educational level $(p=0.001)$; total duration of maternal education $(p=0.001)$; increasing paternal educational level $(p=0.001)$; wealth index $(p=0.001)$; and who the decision maker was regarding the mother's health $(p=0.014)$ (Tables 1 and 2).

Binomial logistic regression was used to identify predictors of appropriate careseeking for suspected pneumonia using the variables listed above that were significant on a univariate analysis. Tertiary level education for the husband/partner (odds ratio (OR) 2.16, $95 \%$ CI 1.03 - 4.52; $p=0.040)$ increased the odds of seeking appropriate care by $116 \%(p=0.040)$. Also belonging to the middle quintile on the wealth index (OR 2.06, 95\% CI 1.12 - 3.80; 
Table 2. Univariate analysis of weighted numbers and percentages of selected determinants of appropriate care-seeking behaviour

\begin{tabular}{|c|c|c|c|}
\hline Variables & $\begin{array}{l}\text { Appropriate, } \\
\boldsymbol{n}(\%)^{\star}\end{array}$ & $\begin{array}{l}\text { Inappropriate, } \\
n(\%)^{*}\end{array}$ & $p$-value \\
\hline Mother's religion & $n=193$ & $n=346$ & 0.230 \\
\hline Islam & $126(65.3)$ & $203(58.7)$ & \\
\hline Christianity & $63(32.6)$ & $138(39.9)$ & \\
\hline Traditionalist & $4(2.1)$ & $5(1.4)$ & \\
\hline Covered by health insurance & $n=192$ & $n=350$ & 0.190 \\
\hline Yes & $4(2.1)$ & $2(0.6)$ & \\
\hline No & $188(97.9)$ & $348(99.4)$ & \\
\hline Household wealth index quintiles & $n=195$ & $n=351$ & $0.0001^{\dagger}$ \\
\hline Poorest & $38(19.5)$ & $98(27.9)$ & \\
\hline Poorer & $52(26.7)$ & $126(35.9)$ & \\
\hline Middle & $46(23.6)$ & $66(18.8)$ & \\
\hline Richer & $27(13.8)$ & $44(12.5)$ & \\
\hline Richest & $32(16.4)$ & $17(4.8)$ & \\
\hline Decision maker regarding health of mother & $n=185$ & $n=326$ & $0.032^{\dagger}$ \\
\hline Mother alone & $8(4.3)$ & $27(8.3)$ & \\
\hline Mother and husband/partner & $65(35.1)$ & $81(24.8)$ & \\
\hline Husband/partner alone & $112(60.5)$ & $216(66.3)$ & \\
\hline Others & $0(0.0)$ & $2(0.6)$ & \\
\hline Current marital status & $n=194$ & $n=350$ & 0.355 \\
\hline Never married & $2(1.0)$ & $13(3.7)$ & \\
\hline Married & $183(94.3)$ & $318(90.9)$ & \\
\hline Living with partner & $2(1.0)$ & $8(2.3)$ & \\
\hline Widowed/separated/divorced & $7(3.6)$ & $11(3.1)$ & \\
\hline
\end{tabular}

$p=0.020)$ and families where decision-making regarding the health of the mother was made jointly by husband/partner and mother (OR 3.64, 95\% CI $1.54-8.61 ; p=0.003)$ are all associated with increased odds of appropriate care seeking for suspected pneumonia. Other parameters failed to achieve significance on the regression model (Tables 3 and 4).

\section{Discussion}

Pneumonia remains the leading infectious cause of death among children under-5, killing $\sim 2600$ children a day. ${ }^{[10]}$ Poor or delayed care accounts for up to $70 \%$ of child deaths in developing countries. ${ }^{[18,19]}$ The occurrence rate for suspected pneumonia among children under 5 years of age in the current study was 19.5 per 1000 children (95\% CI 18.0 - 21.1).

Appropriate case management is a major focus of both the 2009 GAPP and the subsequent integrated Global Action Plan for Pneumonia and Diarrhoea. ${ }^{[6,20]}$ Children with pneumonia should receive a full course of effective antibiotics because most severe cases have a bacterial cause..$^{[2,15,21]}$ Timely institution of appropriate treatment is dependent on early recognition of the symptomatology by parents, decision to seek care from appropriate sources, availability, accessibility and affordability of the necessary therapy. ${ }^{[2]}$ Studies have shown that parents are able to recognise the common symptoms and signs of potentially severe ill health, but that this does not always translate into appropriate health-seeking action. ${ }^{[18,22,23]} \mathrm{In}$ the current study, care-seeking behaviour was poor with only $35.7 \%$ of parents of children with suspected pneumonia seeking care from appropriate sources. This is much lower than the sub-Saharan African average of $48 \%$ and the average for developing countries of $60 \% \cdot{ }^{[2]}$ It is, however slightly higher than the $32 \%$ reported in NDHS 2008. ${ }^{[14]}$ It suggests that care-seeking behaviour for pneumonia in Nigeria has remained almost the same over the 5-year period from 2008 to 2013 . This is in contrast to reports from most other countries that have shown significant improvement in care-seeking behaviour. ${ }^{[2,10]}$
The decision-making process regarding medical care for ill health by parents is complex and often influenced by several factors. ${ }^{[7,10-13]}$ Cultural factors, parental education, previous experience, knowledge and socioeconomic status often influence health-related decisionmaking in developing countries. ${ }^{[7,8,11,13,18]}$ These factors would contribute in differing magnitudes depending on location and culture. The educational level of the husband or partner contributed significantly to appropriate care seeking in this study. A significantly higher proportion $(48 \%)$ of male partners who had sought for appropriate care had secondary school education or more compared with only $32.6 \%$ of those who had inappropriate care. While maternal education was significant on a univariate analysis but did not reach statistical significance on the regression analysis, a cursory look at the data suggests it may still play a role. Possible interactions between maternal and paternal education may have eliminated the role of the mother's education in the regression analysis. Also, considering the husband or partner was responsible for decision-making (alone or in combination with the mother) regarding the health of the mother in $92.8 \%$ of the respondents, it is not surprising that maternal education did not significantly influence appropriate care seeking. This is further strengthened by the finding in this study that where decisions regarding health of the mother are taken by both partners, the odds of seeking appropriate care for the child with suspected pneumonia increase almost three fold. Taffa and Chepngeno ${ }^{[9]}$ demonstrated that maternal education played an important role in determining appropriate care-seeking behaviour for children with diarrhoea, pneumonia and malaria in Kenya. Therefore, we postulate that unlike the findings in our study, maternal education may influence appropriate health decisions in societies where women play a key role in decision-making in the household. The finding that parents in the higher wealth quintile were more likely to seek appropriate care independent of education suggests that the cost of medical care plays a significant role in the decision-making process. This is especially important as the majority of parents in the study relied on out of pocket expenses for care. Obviously parents in the higher wealth quintiles are able to afford these expenses readily and as such would more appropriately seek proper care. Similar findings regarding cost and willingness/ability to pay for appropriate care have been reported by other authors. $^{[24]}$

\section{Study limitation}

A limitation of the study was the use of suspected pneumonia as the factor defining the need for care seeking. Caution must be taken in the use of suspected pneumonia as a defining factor for determining true burden of pneumonia as the 
Table 3. Determinants of appropriate care seeking for children with suspected pneumonia using unweighted data

\begin{tabular}{lll}
\hline Variable & OR $(95 \% \mathrm{CI})^{*}$ & $p$-value \\
\hline Mean (SD) duration of education $(\mathrm{yr})$ & $1.11(0.84-1.48)$ & 0.47 \\
Place of residence & & \\
$\quad$ Urban & 1.00 & \\
Rural & $1.50(0.88-2.57)$ & 0.136 \\
Highest maternal educational level & & \\
None & 1.00 & \\
Incomplete primary & $0.47(0.14-1.60)$ & 0.23 \\
Complete primary & $0.50(0.08-2.99)$ & 0.45 \\
Incomplete secondary & $0.30(0.02-3.95)$ & 0.36 \\
Complete secondary & $0.59(0.02-18.08)$ & 0.76 \\
Tertiary & $0.36(0.00-29.87)$ & 0.65 \\
Highest paternal educational level & & \\
None & 1.00 & \\
Incomplete primary & $1.05(0.40-2.76)$ & 0.93 \\
Complete primary & $1.04(0.55-1.96)$ & 0.91 \\
Incomplete secondary & $1.06(0.51-2.20)$ & 0.87 \\
Complete secondary & $1.20(0.64-2.24)$ & 0.58 \\
Tertiary & $2.16(1.03-4.52)^{\dagger}$ & $0.04^{\dagger}$ \\
*Unweighted data used for regression. & & \\
${ }^{\dagger}$ Statistically significant. & & \\
& &
\end{tabular}

term includes several false positives and as such overestimates pneumonia two to seven fold. ${ }^{[25]}$ However, its use as a denominator for evaluating careseeking behaviour in large-scale studies ( $\geq 20000$ children) is appropriate and has been validated. ${ }^{[25]}$

\section{Conclusion}

The study has strengthened the global recommendation that pneumonia case management efforts must take care-seeking behaviour into account. It has also demonstrated that interventions focusing on improving education, socioeconomic status of parents, empowering women in decision-making and increased coverage of health insurance may play a crucial role in improving pneumonia-related morbidity and mortality in Nigeria.

\section{References}

1. UNICEF. Committing to Child Survival: A Promise Renewed Progress Report 2014. New York: UNICEF, 2014.

2. Gupta G. Tackling pneumonia and diarrhoea: The deadliest diseases for the world's poorest children. Lancet 2012;379(9832):2123-2124. [http://dx.doi. org/10.1016/S0140-6736(12)60907-6]

3. Wardlaw T, You D, Hug L, Amouzou A, Newby H. UNICEF Report: Enormous progress in child survival but greater focus on newborns urgently needed. Reprod Health 2014;11(1):82. [http://dx.doi.org/10.1186/1742-4755-11-82]

4. World Health Organization. Global Action Plan for the Prevention and Control of Pneumonia in children aged under 5 years. Wkly Epidemiol Rec 2009;84(43):451-452. [http://dx.doi.org/10.2471/BLT.08.053348]

5. Qazi S, Weber M, Boschi-Pinto C, Cherian T. Global action plan for the prevention and control of pneumonia (GAPP): Report of an informal consultation: La Mainaz, Gex, France, 5 - 7 March 2007. Geneva: World Health Organization, 2008:1-24.

6. Qazi S, Aboubaker S, MacLean R, et al. Ending preventable child deaths from pneumonia and diarrhoea by 2025. Development of the integrated Global Action Plan for the Prevention and Control of Pneumonia and Diarrhoea. Arch Dis Child 2015;100(Suppl 1):S23-S28. [http://dx.doi.org/10.1136/archdischild-2013-305429]

7. Abdulkadir MB, Ibraheem RM, Johnson WBR. Sociodemographic and clinical determinants of time to care-seeking among febrile children under-five in North-Central Nigeria. Oman Med J 2015;30(5):331-335. [http://dx.doi. org/10.5001/omj.2015.68]
Table 4. Determinants of appropriate care seeking for children with suspected pneumonia using unweighted data

\begin{tabular}{|c|c|c|}
\hline Variable & $\mathrm{OR}(95 \% \mathrm{CI})^{*}$ & $p$-value \\
\hline \multicolumn{3}{|l|}{ Household wealth index quintiles } \\
\hline Poorest & 1.00 & \\
\hline Poorer & $1.14(0.67-1.93)$ & 0.63 \\
\hline Middle & $2.06(1.12-3.80)^{\dagger}$ & $0.02^{\dagger}$ \\
\hline Richer & $1.48(0.67-3.26)$ & 0.34 \\
\hline Richest & $1.13(0.37-3.43)$ & 0.83 \\
\hline \multicolumn{3}{|c|}{$\begin{array}{l}\text { Decision maker regarding health of } \\
\text { mother }\end{array}$} \\
\hline Mother alone & 1.00 & \\
\hline Mother and husband/partner & $3.64(1.54-8.61)^{\dagger}$ & $0.003^{\dagger}$ \\
\hline Husband/partner alone & $2.71(1.17-6.27)^{\dagger}$ & $0.020^{\dagger}$ \\
\hline Others & $0.00(0.00-\infty)$ & 1.00 \\
\hline
\end{tabular}

8. Abdulraheem I, Parakoyi D. Factors affecting mothers' healthcare-seeking behaviour for childhood illnesses in a rural Nigerian setting. Early Child Dev Care 2009;179(5):671-683. [http://dx.doi.org/10.1080/03004430701500885]

9. Taffa N, Chepngeno G. Determinants of health care seeking for childhood illnesses in Nairobi slums. Trop Med Int Health 2005;10(3):240-245. [http:// dx.doi.org/10.1111/j.1365-3156.2004.01381.x]

10. UNICEF. Despite steady progress, pneumonia remains one of the single largest killer of young children worldwide. 2013. http://data.unicef.org/child-health/ pneumonia.html (accessed 11 January 2015).

11. Sreeramareddy C, Shankar R, Sreekumaran B, Subba S, Joshi H, Ramachandran U. Care seeking behaviour for childhood illness: A questionnaire survey in western Nepal. BMC Int Health Hum Rights 2006;6(1):7. [http://dx.doi. org/10.1186/1472-698X-6-7]

12. D’Souza R. Care-seeking behavior. Clin Infect Dis 1999;28(2):234. [http:// dx.doi.org/10.1086/515120]

13. Goldman N, Heuveline P. Health-seeking behaviour for child illness in Guatemala. Trop Med Int Health 2000;5(2):145-155. [http://dx.doi.org/10.1046/ j.1365-3156.2000.00527.x]

14. National Population Commission (Nigeria), ICF International. Nigeria Demographic and Health Survey 2013. Abuja: National Population Commission, 2014.

15. Gove S. Integrated management of childhood illness by outpatient health workers: Technical basis and overview. Bull World Health Organ 1997;75(1):7-16.

16. Rutstein SO, Rojas G. Guide to DHS Statistics. Calverton: ORC Macro, 2006:1-160

17. Rutstein SO, Johnson K. The DHS Wealth Index. Calverton: ORC Macro, 2004.

18. Hill Z, Kendall C, Arthur P, Kirkwood B, Adjei E. Recognizing childhood illnesses and their traditional explanations: Exploring options for care-seeking interventions in the context of the IMCI strategy in rural Ghana. Trop Med Int Health 2003;8(7):668-676. [http://dx.doi.org/10.1046/j.1365-3156.2003.01058.x]

19. de Souza ACT, Peterson KE, Andrade FMO, Gardner J, Ascherio A. Circumstances of post-neonatal deaths in Ceara, Northeast Brazil: Mothers' care-seeking behaviors during their infants' fatal illness. Soc Sci Med 2000;51(11):1675-1693. [http://dx.doi.org/10.1016/S0277-9536(00)00100-3]

20. Greenwood B. A global action plan for the prevention and control of pneumonia. Bull World Health Organ 2008;86(5):322-322A. [http://dx.doi. org/10.1590/S0042-96862008000500002]

21. Sazawal S, Black RE. Effect of pneumonia case management on mortality in neonates, infants, and preschool children: A meta-analysis of communitybased trials. Lancet Infect Dis 2003;3(9):547-556. [http://dx.doi.org/10.1016/ S1473-3099(03)00737-0]

22. Herman E, Black RE, Wahba S, Khallaf N. Developing strategies to encourage appropriate care-seeking for children with acute respiratory infections: An example from Egypt. Int J Health Plann Manage 1994;9(3):235-243.

23. Hildenwall H, Nantanda R, Tumwine JK, et al. Care-seeking in the development of severe community acquired pneumonia in Ugandan children. Ann Trop Paediatr 2009;29(4):281-289. [http://dx.doi.org/10.1179/027249309X12547917869005]

24. Rutebemberwa E, Kallander K, Tomson G, Peterson S, Pariyo G. Determinants of delay in care-seeking for febrile children in eastern Uganda. Trop Med Int Health 2009;14(4):472-479. [http://dx.doi.org/10.1111/j.1365-3156.2009.02237.x]

25. Campbell $\mathrm{H}$, el Arifeen S, Hazir T, et al. Measuring coverage in $\mathrm{MNCH}$ : Challenges in monitoring the proportion of young children with pneumonia who receive antibiotic treatment. PLoS Med 2013;10(5):e1001421.[http:// dx.doi.org/10.1371/journal.pmed.1001421] 